\title{
Potential resurgence of respiratory syncytial virus in Canada
}

\author{
Pascal M. Lavoie MDCM PhD, Frederic Reicherz MD, Alfonso Solimano MD, Joanne M. Langley MD MSc
}

Cite as: CMAJ 2021 July 26;193:E1140-1. doi: 10.1503/cmaj.210919

A ustralia and, more recently, the United States have seen a resurgence of cases of respiratory syncytial virus (RSV) infection in the summer season, as COVID-19 pandemicrelated physical distancing measures are being relaxed. We argue that Canada should anticipate a similar resurgence in seasonal respiratory viruses in the summer of 2021. It is crucial that we continue to monitor respiratory illnesses to inform RSV prevention programs and help protect vulnerable patients. This may involve administering RSV monoclonal antibody therapy in high-risk children off season, which is a major departure from standard practice.

Before the COVID-19 pandemic, lower respiratory tract infection caused an estimated 2.7 million deaths annually worldwide, ranking fourth in cause-specific mortality. ${ }^{1}$ Respiratory syncytial virus and influenza are leading causes of lower respiratory tract infection in young children. In children younger than 2 years, RSV presents as a bronchiolitis and, in younger infants, it presents with nonspecific symptoms like apnea and reduced oral intake. There is no RSV vaccine and no population-level intervention exists beyond basic hygiene measures. In Canada, high-risk children younger than 2 years - including infants born prematurely and those with severe chronic lung disease or serious congenital heart disease - currently receive monthly RSVneutralizing monoclonal antibody therapy during the RSV season (typically late fall to early spring in Canada), ${ }^{2}$ which reduces the risk of hospital admissions related to lower respiratory tract infection by more than half. This occurs annually on fixed dates based on past epidemiology, or on a variable season start based on active surveillance.

Since the beginning of the pandemic, many countries have observed a near total disappearance of RSV and influenza cases., In Canada, 339627 tests for RSV were reported through the Centre for Immunization and Respiratory Infectious Diseases. Of these tests, 239 were positive between Aug. 29, 2020, and May 8, 2021. In comparison, 412861 tests for RSV were reported over a similar period in the previous year (Aug. 25, 2019, and May 2, 2020), of which 18860 were positive. In response to the apparent disappearance of RSV during the pandemic, several Canadian RSV programs paused, shortened and even suspended the seasonal administration of monoclonal antibody, to avoid unnecessary medical visits. ${ }^{6}$ However, an interseason resurgence of RSV cases has recently been reported in the southern hemisphere in the setting of decreased SARS-CoV-2 circulation and relaxed physical distancing measures. ${ }^{7,8}$ The US Centers for Disease Control and Prevention

\section{KEY POINTS}

- Australia and, more recently, the United States have seen a resurgence of cases of respiratory syncytial virus (RSV) infection in parallel with reduced pandemic-related physical distancing measures.

- Canada should also anticipate a similar resurgence and should monitor respiratory illness to be able to quickly respond with strong RSV prevention measures.

- For many months, Canada has seen virtually no cases of RSV infection, which may mean that pregnant women and infants have had lower exposure and therefore pediatric immunity levels may be low.

- A resurgence in cases in the summer of 2021 could stretch resources in pediatric intensive care units across Canada.

- Respiratory syncytial virus programs should prepare to administer immunoprophylaxis off season, to the highest-risk infants.

also issued a health alert on June 10, 2021, about increased interseasonal RSV activity in the southern US since March 2021 (https://emergency.cdc.gov/han/2021/han00443.asp). The offseason resurgence in seasonal respiratory viruses now potentially poses a threat to vulnerable infants.

The resurgence of cases of RSV in Australia and the US highlights the importance of population immunity in regulating RSV epidemics after exposure peaks. Studies have documented cyclical variations in maternally derived antibody levels, with an increase during the RSV winter season, followed by a decline over the summer months. ${ }^{9}$ During RSV seasons, most affected adults younger than 65 years remain asymptomatic or develop mild illness, owing to a lifelong seasonal exposure to the virus and memory B- and T-cell immunity. In contrast, immunologically naive infants depend on passively transferred maternal antibodies to protect them at birth, but these antibodies wane within 6 months. Asymptomatic testing in households indicates that transmission may have continued to occur undetected during the COVID-19 pandemic, ${ }^{10}$ but it is more likely that during the pandemic, pregnant individuals were less likely to be exposed to RSV, and thus less likely to boost their RSV antibodies to levels usually seen in the winter. This raises a possibility that infants are less well protected than usual and could become sicker if they are infected this summer. 
In Australia, during the recent re-emergence of RSV, the median age of children in whom RSV testing was positive increased to 18.4 months, which was higher than the upper range between 2012 and 2019 (7.3-12.5 months; $p<0.001) .{ }^{7}$ This could suggest that infants who were not exposed to RSV in their first year did not develop sufficient immunity, such that they remained susceptible into their second year. Pediatric intensivists in Australia reported an increase in severe, atypical RSV cases in the first 4 months of 2021 (end of summer, early autumn in the months after the lockdown) in older children requiring mechanical ventilation (Trevor Duke, Paediatrics Royal Children's Hospital, Melbourne, Australia, personal communication). If a similar phenomenon happened in Canada, the burden of illness could stretch resources in pediatric intensive care units (ICUs) during the summer of 2021. Although we have been unable to find published data on changes in public health responses to the offseason surge in RSV and influenza cases, we are aware that some jurisdictions in Australia started monoclonal therapy early and decided to extend RSV immunoprophylaxis until cases subsided.

In anticipation of a possible resurgence of RSV in Canada, we suggest the following approach. First, as pandemic-related physical distancing measures are relaxed, health care providers should continue to emphasize basic hygiene measures such as washing hands. We support ongoing efforts to keep vulnerable infants from coming into contact with people with respiratory illness. Other protective measures like breastfeeding when possible and avoiding exposure to second-hand smoke should be continued and emphasized. Second, confirmatory RSV testing should continue, following usual indications, to provide surveillance data. Third, pediatric ICUs should anticipate an increase in severe RSV cases, as reported in Australia. ${ }^{7}$ Respiratory syncytial virus programs should prepare to administer immunoprophylaxis off season, to the highest-risk infants, if cases increase to levels that normally trigger the fall season start.

In Canada, RSV programs have not yet prospectively defined how many cases of RSV infection would constitute a resurgence, but it seems reasonable to consider that even a few cases should trigger communications with stakeholders, particularly considering that for months we have seen 0 cases every week in many jurisdictions. Nonetheless, it is worth mentioning that whether the use of RSV monoclonal therapy off season is cost-effective in this context is unclear. We do not anticipate drug shortages, but new arrangements may need to be coordinated for shipments and storage of palivizumab, in preparing for a resurgence of RSV, earlier than usual. Finally, studies are needed to document the impact of the pandemic on the cyclical occurrence of seasonal viruses and to understand why RSV and influenza disappeared in the past year, while SARS-CoV-2 and rhinoviruses continued to circulate. ${ }^{11}$

\section{References}

1. GBD 2015 Mortality and Causes of Death Collaborators. Global, regional, and national life expectancy, all-cause mortality, and cause-specific mortality for 249 causes of death, 1980-2015: a systematic analysis for the Global Burden of Disease Study 2015. Lancet 2016;388:1459-544.

2. Obando-Pacheco P, Justicia-Grande AJ, Rivero-Calle I, et al. Respiratory syncytial virus seasonality: a global overview. J Infect Dis 2018;217:1356-64.

3. Yeoh DK, Foley DA, Minney-Smith CA, et al. The impact of COVID-19 public health measures on detections of influenza and respiratory syncytial virus in children during the 2020 Australian winter. Clin Infect Dis 2020;72:2199-2202.

4. Rodgers L, Sheppard M, Smith A, et al. Changes in seasonal respiratory illnesses in the United States during the COVID-19 pandemic. Clin Infect Dis 2021 Apr 29 [Epub ahead of print]; ciab311. doi: 10.1093/cid/ciab311.

5. Respiratory virus detections in Canada. Ottawa: Public Health Agency of Canada; 2021. Available: https://www.canada.ca/en/public-health/services/surveillance/ respiratory-virus-detections-canada.html (accessed 2021 May 12).

6. Taylor RS. Respiratory syncytial virus and palivizumab prophylaxis in the COVID-19 era [letter]. CMAJ 2021;193:E523.

7. Foley DA, Yeoh DK, Minney-Smith CA, et al. The interseasonal resurgence of respiratory syncytial virus in Australian children following the reduction of coronavirus disease 2019-related public health measures. Clin Infect Dis 2021 Feb 17 [Epub ahead of print]; ciaa1906. doi: 10.1093/cid/ciaa1906.

8. McNab S, Ha Do LA, Clifford V, et al. Changing epidemiology of respiratory syncytial virus in Australia - delayed re-emergence in Victoria compared to WA/ NSW after prolonged lock-down for COVID-19. Clin Infect Dis 2021 Mar 18 [Epub ahead of print];ciab240. doi: 10.1093/cid/ciab240.

9. Stensballe LG, Ravn H, Kristensen K, et al. Seasonal variation of maternally derived respiratory syncytial virus antibodies and association with infant hospitalizations for respiratory syncytial virus. J Pediatr 2009;154:296-8.

10. Emanuels A, Heimonen J, O'Hanlon J, et al. Remote household observation for non-influenza respiratory viral illness. Clin Infect Dis 2020 Nov 17; ciaa1719 [Epub ahead of print]. doi: 10.1093/cid/ciaa1719.

11. Baker RE, Park SW, Yang W, et al. The impact of COVID-19 nonpharmaceutical interventions on the future dynamics of endemic infections. Proc Natl Acad Sci U S A 2020;117:30547-53.
Competing interests: Pascal Lavoie and Alfonso Solimano serve on the Executive Committee, and Alfonso Solimano is the Medical Director, of the British Columbia Respiratory Syncytial Virus (RSV) Immunoprophylaxis Program, a publicly funded program under the British Columbia Ministry of Health's Provincial Health Services Authority. Joanne Langley's study site has done research studies on RSV vaccines with GSK, Janssen and Pfizer. Funding for these studies is paid to Dalhousie University. No other competing interests were declared.

This article has been peer reviewed.

Affiliations: Division of Neonatology, Department of Pediatrics (Lavoie, Solimano), University of British Columbia; BC Children's Hospital Research Institute (Lavoie, Reicherz), Vancouver, BC; Canadian Center for Vaccinology, Dalhousie University (Langley), IWK Health and Nova Scotia Health, Halifax, NS

Contributors: All of the authors contributed to the conception and design of the work, drafted the manuscript, revised it critically for important intellectual content, gave final approval of the version to be published and agreed to be accountable for all aspects of the work.

Content licence: This is an Open Access article distributed in accordance with the terms of the Creative Commons Attribution (CC BY-NC-ND 4.0) licence, which permits use, distribution and reproduction in any medium, provided that the original publication is properly cited, the use is noncommercial (i.e., research or educational use), and no modifications or adaptations are made. See: https://creativecommons.org/ licenses/by-nc-nd/4.0/

Acknowledgements: The authors thank Dr. Manish Sadarangani and David Goldfarb and Joan Robinson for ongoing, insightful discussions on this issue, and for their expert input on this commentary, and Cheryl Christopherson for surveying provincial RSV programs in Canada on practices during the pandemic.

Correspondence to: Pascal Lavoie, plavoie@cw.bc.ca 